\title{
Desarrollo del cladócero nativo Ceriodaphnia sp con clorofitas (Scenedesmus sp y Chlorella sp) en condiciones de laboratorio
}

\author{
Growth of the native cladocero Ceriodaphnia sp with chlorophytes (Scenedesmus \\ sp and Chlorella sp) under laboratory conditions
}
Rosa Angélica Ismiño Orbe ${ }^{1,3}$, Jenifer Jazmín Araujo-Solis ${ }^{2}$, Séfora Mishell Vargas- Del Castillo ${ }^{2}$, Kevin Morgan Ruiz-Tafur ${ }^{2}$, Humberto Arbildo-Ortiz ${ }^{2}$, Miriam Adriana Alvan-Aguilar ${ }^{1}$

\section{Resumen}

\begin{abstract}
Se presentan los resultados del efecto de las clorofitas Scenedesmus sp y Chlorella sp utilizadas como alimento sobre el desarrollo del cladócero nativo Ceriodaphnia sp. Se emplearon tres tratamientos con tres repeticiones para cada clorifita, $\mathrm{T} 1=5 \mathrm{ml} . \mathrm{L}^{-1}$ $\left(25 \times 10^{6} \mathrm{cel}\right), \mathrm{T} 2=10 \mathrm{ml} \cdot \mathrm{L}^{-1}\left(50 \times 10^{6} \mathrm{cel}\right)$ y T3 $=15 \mathrm{ml} \cdot \mathrm{L}^{-1}\left(75 \times 10^{6} \mathrm{cel}\right)$. Las características físicas y químicas del agua de los recipientes variaron entre 28.4 y $28.6^{\circ} \mathrm{C}$, pH entre 6.57 y 7.36 y el oxígeno disuelto entre 6.34 y $6.66 \mathrm{mg} / \mathrm{l}$ entre tratamientos. Los resultados mostraron que Ceriodaphnia sp tiene preferencia por la microalga Chlorella sp, donde las dietas con la concentración de $10 \mathrm{ml}^{-1^{-1}}\left(50 \times 10^{6} \mathrm{cel}\right)$ fueron superiores a las otras dietas.
\end{abstract}

Palabras clave: cladócero, Ceriodaphnia sp, clorofitas, cultivo

\section{AbSTRaCT}

The effect of the chlorophytes Scenedesmus sp and Chlorella sp used as feed on the growth of the native cladoceran Ceriodaphnia sp were studied. Three treatments with three repetitions for each chlorophyte were used: T1 $=5 \mathrm{ml} . \mathrm{L}-1\left(25 \times 10^{6}\right.$ cells $), \mathrm{T} 2=$ $10 \mathrm{ml} . \mathrm{L}-1\left(50 \times 10^{6}\right.$ cells $)$ and T3 $=15 \mathrm{ml} . \mathrm{L}-1\left(75 \times 10^{6} \mathrm{cel}\right)$. The physical and chemical characteristics of the water in the containers varied between 28.4 and $28.6^{\circ} \mathrm{C}$, pH between

\footnotetext{
${ }^{1}$ Programa de Investigación para el Uso del Agua y Sus Recursos (AQUAREC), Instituto de Investigaciones de la Amazonia Peruana (IIAP), Iquitos, Perú

${ }^{2}$ Facultad de Ciencias Biológicas, Universidad Nacional de la Amazonia Peruana, Iquitos, Perú

${ }^{3}$ E-mail: rayo14@hotmail.com
} 
6.57 and 7.36 and the dissolved oxygen between 6.34 and $6.66 \mathrm{mg} / \mathrm{l}$ between treatments. The results showed that Ceriodaphnia sp prefers the Chlorella sp microalgae, where diets with a concentration of $10 \mathrm{ml} .1-1\left(50 \times 10^{6}\right.$ cells $)$ were better than the other diets.

Key words: cladocero, Ceriodaphnia sp, chlorophytes, culture

\section{INTRODUCCIÓN}

El empleo de fitoplancton y zooplancton como alimento vivo de larvas y alevinos de peces se ha convertido en una opción alimenticia y económica que asegura la sobrevivencia de los peces en las etapas iniciales de vida. Estos organismos planctónicos contienen proteínas, carbohidratos, ácidos grasos, aceites esenciales y enzimas, como proteinasas, pepsidasas y amilasas entre otras, que aseguran una adecuada nutrición y alimentación para las larvas de peces (SipaúbaTavares y Rocha, 2003). La producción de fitoplancton y zooplancton es una actividad rentable y de poca inversión, por lo que muchos centros de producción de semilla de peces vienen incorporando la tecnología de cultivo de estos organismos como una alternativa alimenticia al uso de quistes de Artemia salina, que es un producto importado y costoso (Velu y Munuswany, 2003).

Dentro del grupo de organismos zooplanctónicos, los cladóceros se destacan por su potencialidad nutritiva para larvas de peces que está en función directa con el sustrato donde se desarrollan (Muñoz, 2006); sin embargo, su producción requiere mantener un crecimiento sostenido. Existen varios estudios enfocados a la búsqueda de un alimento que permita el crecimiento sostenido de los cladóceros, entre ellos resalta el uso de microalgas y levaduras (Prieto et al., 2006). Los cladóceros, por ser filtradores no selectivos, se pueden alimentar de ambos ti- pos de alimentos a la vez (Romero, 2009). La combinación de microalgas + levaduras, así como de varias especies de microalgas han mostrado buenos resultados en el crecimiento de cladóceros (Santos et al., 2006; Prieto y Atencio 2008; Ocampo et al., 2010).

Un estudio de Moina sp alimentado con Ankistrodesmus sp y Ankistrodesmus sp + Saccharomyces cerevisiae encontraron mejor resultado en el crecimiento con la mezcla de dichos alimentos (Prieto et al., 2006). Peña-Aguado et al. (2005) evaluaron el crecimiento de Ceriodaphnia dubia y Moina macrocopa alimentados con Chlorella vulgaris, Scenedesmus acutus y Saccharomyces cerevisiae y obtuvieron los mejores resultados con las combinaciones de las dos microalgas, mientras que Jiménez et al. (2003) en Moina macrocopa, estudiando el efecto de diferentes fuentes de alimento como Scenedesmus abundans y Saccharomyses cerevisiae y alimento artificial Z-plus para protozoos de camarones marinos, encontraron mayor densidad con este último. El cladócero nativo Ceriodaphnia es un género frecuente y común que se encuentra en ecosistemas dulceacuícolas tropicales y subtropicales, y se distribuye ampliamente debido a su tolerancia a variaciones de temperatura (Villalobos y González, 2005).

El objetivo del presente trabajo fue evaluar el efecto de dos microalgas (Scenedesmus y Chlorella) como alimento sobre el desarrollo del cladócero nativo Ceriodaphnia sp de la Amazonia peruana. 


\section{Materiales y Métodos}

\section{Área de Estudio}

El presente estudio fue realizado en el Laboratorio de Cultivo Auxiliares del Centro de Investigaciones Fernando Alcántara Bocanegra (CIFAB) del Instituto de Investigaciones de la Amazonia Peruana - IIAP, ubicado en el km 4.5 de la Carretera IquitosNauta, Loreto-Perú.

\section{Colecta y Aislamiento}

Las muestras del cladócero Ceriodaphnia sp y de las clorofitas (Chlorella sp y Scenedesmus sp) fueron colectadas en los estanques del CIFAB, utilizando una red planctónica con abertura de $30 \mu \mathrm{m}$, donde se filtraron $50 \mathrm{~L}$ de agua. Las muestras fueron colocadas en frascos de polietileno de $500 \mathrm{ml}$ de capacidad y transportadas al laboratorio de cultivos auxiliares, donde fueron identificadas y aisladas con ayuda de un microscopio Leica DM 750. Para la identificación de Ceriodaphnia sp se utilizaron las claves dicotómicas de EL-Moor (1997) y Pennak (1978) y para el aislamiento se empleó la técnica de la pipeta capilar. Para la identificación de las microalgas se utilizó la clave taxonómica de Bicudo y Menezes (2005) y el aislamiento se hizo mediante la técnica de rayado en placas y pipeta capilar.

\section{Obtención de Cepas}

La cepa de cada microalga (Chlorella sp y Scenedesmus sp) fue obtenida utilizando como medio de cultivo un fertilizante agrícola (Byfoland: NPK -11-8-6) a razón de $0.2 \mathrm{ml} / 1$. En el caso de los cladóceros Ceriodaphnia sp fueron seleccionados aleatoriamente y colocados en un frasco Erlenmeyer de $1 \mathrm{~L}$, utilizándose solo $500 \mathrm{ml}$ de agua filtrada esterilizada y con una densidad inicial de 1 individuo x $100 \mathrm{ml}$.

\section{Diseño Experimental}

Se trabajó con tres tratamientos de alimentación del cladócero Ceriodapnia sp,

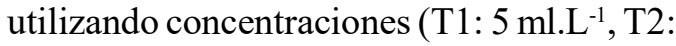
$10 \mathrm{ml} . \mathrm{L}^{-1}$, T3: $15 \mathrm{ml} . \mathrm{L}^{-1}$ ) de cada microalga (Scenedesmus sp y Chlorella sp) (Cuadro 1). Para cada tratamiento se realizó un recuento celular aplicando la técnica de Pérez (1995), utilizando una cámara de Neubauer y un microscopio óptico (Leica ICC50 HD) con aumento de 40x. Durante el experimento se contó con aireación constante y luz las 24 horas del día. Se realizaron tres réplicas por tratamiento y dos ensayos para cada clorofita. El periodo de evaluación fue de 17 días para cada ensayo.

\section{Conteo del Cladócero}

El recuento de Ceriodapnia sp se hizo cada cuatro días utilizando la cámara de Sedgewick- Rafter, siguiendo el procedimiento descrito por Galván (1982). Se analizaron las siguientes variables poblacionales: Crecimiento Poblacional ( $\mathrm{Ind} / \mathrm{ml})$, Tasa Instantánea de Crecimiento (TCE) $\left(\right.$ días $\left.^{-1}\right)$, Tiempo de Duplicación (TD) (días) y Rendimiento (R) (Ind.ml.día), siguiendo los criterios reportados por Prieto et al. (2006) y Heugens et al. (2006).

\section{Parámetros Fisicoquímicos}

Los parámetros físicos y químicos de temperatura, $\left({ }^{\circ} \mathrm{C}\right)$ oxígeno disuelto $(\mathrm{mg} / \mathrm{l})$ y $\mathrm{pH}$ del agua se evaluaron cada dos días. La temperatura y el oxígeno disuelto fueron medidos con un oxímetro digital (YSY 550) y el $\mathrm{pH}$ utilizando un $\mathrm{pH}$ metro digital (WWTpH3210).

\section{Análisis Estadístico}

Se empleó el Diseño Completamente al Azar y se utilizó una hoja de cálculo Excel (Microsoft Office 2010) para la base de da- 

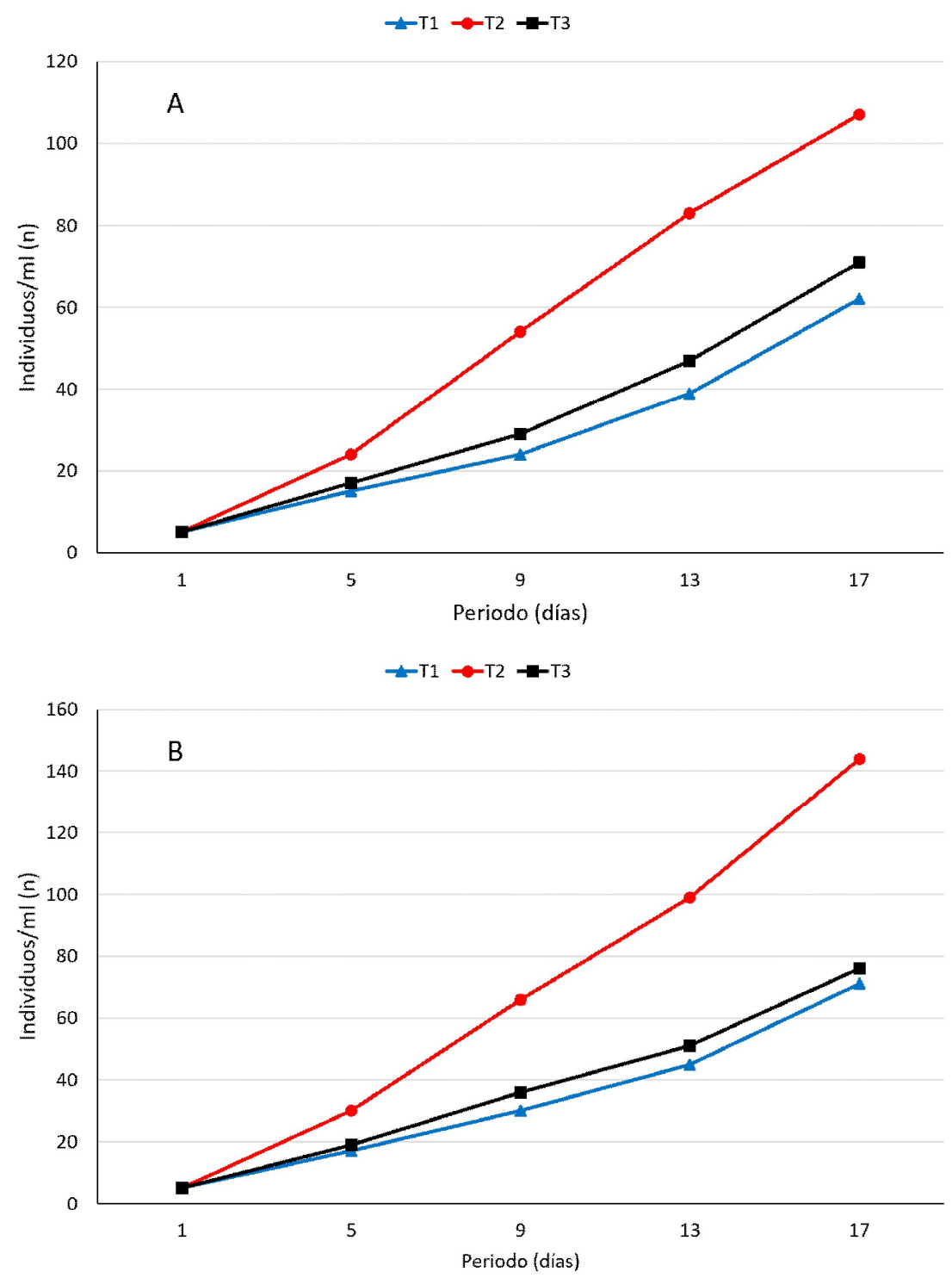

Figura 1. Crecimiento de Ceriodaphnia sp alimentados con (A) Scenedesmus sp y (B) Chlorella sp en condiciones de laboratorio

tos del crecimiento poblacional y de los parámetros fisicoquímicos. El análisis descriptivo se hizo mediante tablas y figuras, empleando el programa BioEstat v. 5.0. Los resultados comparativos fueron analizados aplicando el análisis de varianza de una vía a un nivel de significancia de 0.05 . Adicionalmente se realizó la prueba de Análisis Principales para relacionar los parámetros fisicoquímicos con el crecimiento de la Ceriodaphnia sp.

\section{Resultados y Discusión}

\section{Crecimiento Poblacional}

Ceriodapnia sp presentó una fase de aclimatación durante los primeros cinco días del experimento. El crecimiento poblacional tuvo luego un comportamiento exponencial, llegando a obtenerse entre 62 y 107 indivi- 
Cuadro 1. Tratamientos para el cultivo de Ceriodaphnia sp (5 individuos/500 $\mathrm{ml}$ de agua) alimentados con Chlorella sp y Scenedesmus sp bajo condiciones de laboratorio

\begin{tabular}{lccc}
\hline Microalga & $\begin{array}{c}\text { Trata- } \\
\text { miento }\end{array}$ & $\begin{array}{c}\text { Concen- } \\
\text { tración } \\
\left(\mathrm{ml}^{-L^{-1}}\right)\end{array}$ & $\begin{array}{c}\text { Recuento } \\
\text { celular } \\
\left(\times 10^{6} \mathrm{cel}\right)\end{array}$ \\
\hline Chlorella sp & T1 & 5 & 25 \\
& T2 & 10 & 50 \\
Scenedesmus sp & T3 & 15 & 75 \\
& T1 & 5 & 25 \\
& T2 & 10 & 50 \\
& T3 & 15 & 75 \\
\hline
\end{tabular}

duos a los 17 días con Scenedesmus sp y entre 71 y 144 individuos con Chlorella sp. En ambos experimentos, el T2 (10 ml. $\left.\mathrm{L}^{-1}\right)$ presentó el mayor crecimiento poblacional (Figuras 1A, 1B). No obstante, la alimentación con Chlorella sp presentó un mejor rendimiento en comparación con la alimentación con Scenedesmus sp, debido probablemente a los efectos de la densidad. Al respecto, Espinoza et al. (1992) y Duncan (1989) indican que la densidad de alimento puede ser desfavorable en la sobrevivencia de cladóceros al afectar su proceso de filtración. De igual forma el alimento limitado conduce a la disminución del crecimiento coincidiendo con los resultados del presente estudio.

El mayor crecimiento de Ceriodaphnia sp alimentados con Chlorella sp coincide con Martínez (1999) trabajando con Ceriodahnia dubia y con clorofitas, entre ellas Chlorella. Así mismo, Nandini y Sarma (2003) señalan que cultivando Ceriodaphnia sp con Chlorella sp satisfacen sus requerimientos nutricionales e incrementan su desarrollo.

En contraste, las microalgas Scenedesmus sp y Chlorella sp favorecen la sobrevivencia de Ceriodaphnia sp. Estas diferencias puedan deberse a la calidad nutricional del alimento suministrado. Una estrategia de vida consiste en invertir su energía en sobrevivencia, por lo tanto, no todas las especies de microalgas proporcionadas como alimento son aprovechadas por todas las especies de cladóceros. Es importante tener en cuenta el tipo y concentración de alimento suministrado y la especie de cladócero (Pérez et al., 2015). Ceriodaphnia sp tiene preferencia por Chlorella sp, lo cual es fácilmente explicado en base a la mayor palatabilidad que ofrece en relación otras microalgas (Martínez, 1999).

Tasa Instantánea de Crecimiento (TCE), Tiempo de Duplicación (TD) y Rendimiento $(\mathbf{R})$

Los valores de TCE variaron entre 0.15 y 0.20 días, resultado inferior a los 0.57 a 0.35 d reportados en Moina micrura por Amarasinghe et al. (1997), pero similar a los 0.13 y $0.15 \mathrm{~d}$ reportados por Otero et al. (2013) trabajando con Diaphanosoma sp y Alona sp, respectivamente. Estos valores bajos podrían atribuirse a la baja densidad poblacional inicial (5 individuos), ya que la mayoría de los trabajos utilizan mayores densidades de siembra.

El tipo de dieta presentó diferencias entre los tratamientos $(\mathrm{p}<0.05)$ con referencia a la densidad poblacional, tasa de crecimiento y rendimiento en el cultivo. Los valores poblacionales fueron más altos en los individuos alimentados con Chlorella sp, presentando mayor TC $(0.20 \pm 0.01)$, menor TD $(3.51 \pm 0.03)$ y mayor $\mathrm{R}(8.19 \pm 0.25$ ind.ml.día) (Cuadro 2).

En este trabajo, el mayor valor encontrado en el T2 fue con la microalga Chlorella sp en la concentración de $50 \times 10^{6} \mathrm{cel}$, resultado que concuerda con el trabajo de Romero et al. (2010) quién obtuvo densidades máximas en Moina sp alimentada con Chlorella sp. Sin embargo, Karlsson y Bystrom (2005) obtuvieron menor tiempo de vida con Ceriodaphnia rigaudi alimentados solo con Chlorella. Es posible que la duración de vida 
Cuadro 2. Parámetros poblacionales de Ceriodaphnia sp alimentadas con Chlorella sp y Scenedesmus sp (TCE: Tasa instantánea de crecimiento; TD: Tiempo de duplicación R: Rendimiento)

\begin{tabular}{clccc}
\hline Tratamientos & Dieta alimentaria & TCE & TD & R \\
\hline \multirow{2}{*}{ T1 } & Scenedesmus sp & $0.15 \pm 0.00^{\mathrm{a}}$ & $4.68 \pm 0.08^{\mathrm{a}}$ & $3.36 \pm 0.16^{\mathrm{a}}$ \\
& Chlorella $\mathrm{sp}$ & $0.16 \pm 0.01^{\mathrm{a}}$ & $4.46 \pm 0.11^{\mathrm{a}}$ & $3.85 \pm 0.26^{\mathrm{a}}$ \\
\multirow{2}{*}{$\mathrm{T} 2$} & Scenedesmus $\mathrm{sp}$ & $0.18 \pm 0.00^{\mathrm{b}}$ & $3.86 \pm 0.09^{\mathrm{b}}$ & $5.98 \pm 0.47^{\mathrm{b}}$ \\
& Chlorella $\mathrm{sp}$ & $0.20 \pm 0.01^{\mathrm{a}}$ & $3.51 \pm 0.03^{\mathrm{b}}$ & $8.19 \pm 0.25^{\mathrm{b}}$ \\
\multirow{2}{*}{ T3 } & Scenedesmus $\mathrm{sp}$ & $0.16 \pm 0.01^{\mathrm{a}}$ & $4.47 \pm 0.12^{\mathrm{b}}$ & $3.88 \pm 0.25^{\mathrm{a}}$ \\
& Chlorella $\mathrm{sp}$ & $0.16 \pm 0.00^{\mathrm{a}}$ & $4.33 \pm 0.09^{\mathrm{a}}$ & $4.19 \pm 0.24^{\mathrm{a}}$ \\
\hline
\end{tabular}

Cuadro 3. Condiciones físicas y químicas registradas en el cultivo de Ceriodaphnia sp en condiciones de laboratorio durante los 17 días de la fase experimental

\begin{tabular}{lcccccc}
\hline & \multicolumn{3}{c}{ Scenedesmus $\mathrm{sp}$} & \multicolumn{3}{c}{ Chlorella sp } \\
\cline { 2 - 7 } & $\mathrm{T} 1$ & $\mathrm{~T} 2$ & $\mathrm{~T} 3$ & $\mathrm{~T} 1$ & $\mathrm{~T} 2$ & $\mathrm{~T} 3$ \\
\hline Temperatura & $28.63 \pm 0.34$ & $28.68 \pm 0.34$ & $28.65 \pm 0.34$ & $28.42 \pm 0.34$ & $28.54 \pm 0.35$ & $28.63 \pm 0.32$ \\
$\mathrm{pH}$ & $6.62 \pm 0.42$ & $6.92 \pm 0.32$ & $7.36 \pm 0.18$ & $6.57 \pm 0.30$ & $6.94 \pm 0.23$ & $7.27 \pm 0.24$ \\
$\begin{array}{l}\text { Oxígeno } \\
\text { disuelto }\end{array}$ & $6.62 \pm 0.46$ & $6.66 \pm 0.26$ & $6.61 \pm 0.42$ & $6.34 \pm 0.24$ & $6.51 \pm 0.14$ & $6.61 \pm 0.18$ \\
\hline
\end{tabular}

de Ceriodaphnia se vea afectada por el tipo de dieta, donde el régimen alimenticio con Chlorella ofrece un alcance positivo a su supervivencia. Los resultados indican que Ceriodaphnia encontró en Chlorella sp la mejor dieta para su supervivencia, desarrollo y reproducción, posiblemente debido a los mayores contenidos de lípidos si se compara con los resultados con Scenedesmus (Kumar y Hwang, 2008).

\section{Condiciones Fisicoquímicas del Cultivo}

Los resultados de las condiciones fisicoquímicas del agua de cultivo se presentan en el Cuadro 3. La temperatura promedio varió entre 28.42 y $28.68^{\circ} \mathrm{C}$, el pH entre 6.57 y 7.36 , y el oxígeno disuelto entre 6.34 y $6.66 \mathrm{mg} / 1$, entre tratamientos. Jiménez et al.
(2003) indica que la temperatura puede afectar el crecimiento y la reproducción en cladóceros, siendo la temperatura óptima entre 24 y $31^{\circ} \mathrm{C}$. Los valores de este trabajo estuvieron dentro del rango considerado como óptimo.

En el análisis de componentes principales (Figura 2), el primer componente (Axis 1) representa el $50.91 \%$ de la variabilidad de los tres tratamientos con sus respectivas réplicas sobre el crecimiento de Ceriodaphnia sp en condiciones de laboratorio, mientras que el segundo componente (Axis 2) lo hizo en un $25.29 \%$. El Axis 1 y Axis 2 representan las variables de calidad de agua ( $\mathrm{pH}$, temperatura y oxígeno), los tratamientos con sus réplicas correspondientes y la variable explicativa índice de crecimiento de Ceriodapnia sp. Se observó que la temperatura está 
correlacionada positivamente con la variable índice de Ceriodaphnia. En este sentido, Rodríguez et al. (2003) y Sipaúba-Tavares y Brachion (2002) consiguieron los mejores resultados para los cladóceros Diaphanosoma birgei y Moina micrura con una temperatura en torno a $25^{\circ} \mathrm{C}$ y dietas a base de microalgas. Por el contrario, los valores de $\mathrm{pH}$ están correlacionados negativamente con el índice de crecimiento del cladócero.

El pH obtenido (6.57-7.36) concuerdan con los reportes de Prieto et al. (2006) y Romero (2009), que trabajaron con Moina sp alimentados con clorofitas y $S$. cereviseae, respectivamente, y puede atribuirse que estos organismos requieren en su alimentación la presencia de ácidos grasos esenciales, tales como ácidos grasos insaturados y polinsaturados. Los valores de oxígeno disuelto (OD) del agua no fueron limitantes para el cultivo de Ceriodaphnia, ya que estos organismos toleran ambientes bajos de oxígeno gracias a la capacidad de sintetizar hemoglobina (Rottman et al., 2003).

\section{Literatura Citada}

1. Amarasinghe BP, Boersma $M$, Vijverberg $J$. 1997. The effect of temperature, and food quantity and quality on the growth and development rates in laboratory-cultured copepods and cladocerans from Sri Lankan reservoir. Hydrobiologia 350: 131-144.

2. Bicudo C, Menezes M. 2005. Gêneros de algas de águas continentais do Brasil chave para identificacão e descricões. Brasil: RIMA. 489 p.

3. Duncan A. 1989. Food limitation and body size in the life cycles of planktonic rotifers and cladocerans. Hydrobiologia 186: 11-28.

4. EL-Moor L. 1997. Manual de identificación de cladóceros limnicos de Brasil. Brasilia: Universa p 1-156.

5. Espinoza CF, Martínez F, Ramírez $R$. 1992. Filtration rate and culture of Moina macropopa (Crustacea:
Cladocera) fed with Scenedesmus incrassatulus (Chlorophyceae) and digested cow manure. An Inst Cienc del Mar Limnol 19: 137-142.

6. Galván M. 1982. Calibración y uso del equipo para recuento de plancton. En: Manual de técnicas de muestreo y análisis de plancton y perifiton. México. $\mathrm{p}$ 93-101.

7. Heugens EH, Tokkie LT, Kraak MH, Van SN, Admiraal W. 2006. Population growth of Daphnia magna under multiple stress conditions: joint effects of temperature, food and cadmium. Environ Toxicol Chem 25: 1399-1407. doi: 10.1897/05-294r.1

8. Jiménez D, Rosas J, Velásquez A, Millán J, Cabrera T. 2003. Crecimiento poblacional y algunos aspectos biológicos del cladócero Moina macrocopa (Straus, 1820) (Brachiopoda, Anomopoda) alimentado con tres dietas en tres salinidades diferentes. Ciencia 11: 22-30.

9. Karlsson J, Bystrom P. 2005. Littoral energy mobilization dominates energy supply for top consumers in subarctic lakes. Limnol Oceanogr 50: 538-543. doi: 10.4319/1o.2005.50.2.0538

10. Kumar R. Hwang S. 2008. Ontogenetic shifts in the ability of the cladoceran, Moina macrocopa straus and Ceriodaphnia cornuta Sars to utilize ciliated protest as food source. Int Rev Hydrobiol 93: 284-296. doi: 10.1002/ iroh.200711013

11. Martínez G. 1999. Estrategias de alimentación de tres especies del zooplancton límnico (Cladócera). Rev Chil Hist Nat 72: 671-676.

12. Muñoz ME. 2006. Alimento vivo para peces. Rev Fac Cienc Básicas 2: 43-63.

13. Nandini S, Sarma SS. 2003. Population growth of some genera of cladocerans in relation to algal food (Chlorella vulgaris) levels. Hydrobiologia 491: 211-219.

14. Ocampo LE, Botero MR, Restrepo LF. 2010. Evaluación del crecimiento de un cultivo de Daphnia magna alimentado con Saccharomyces cereviseae y un 


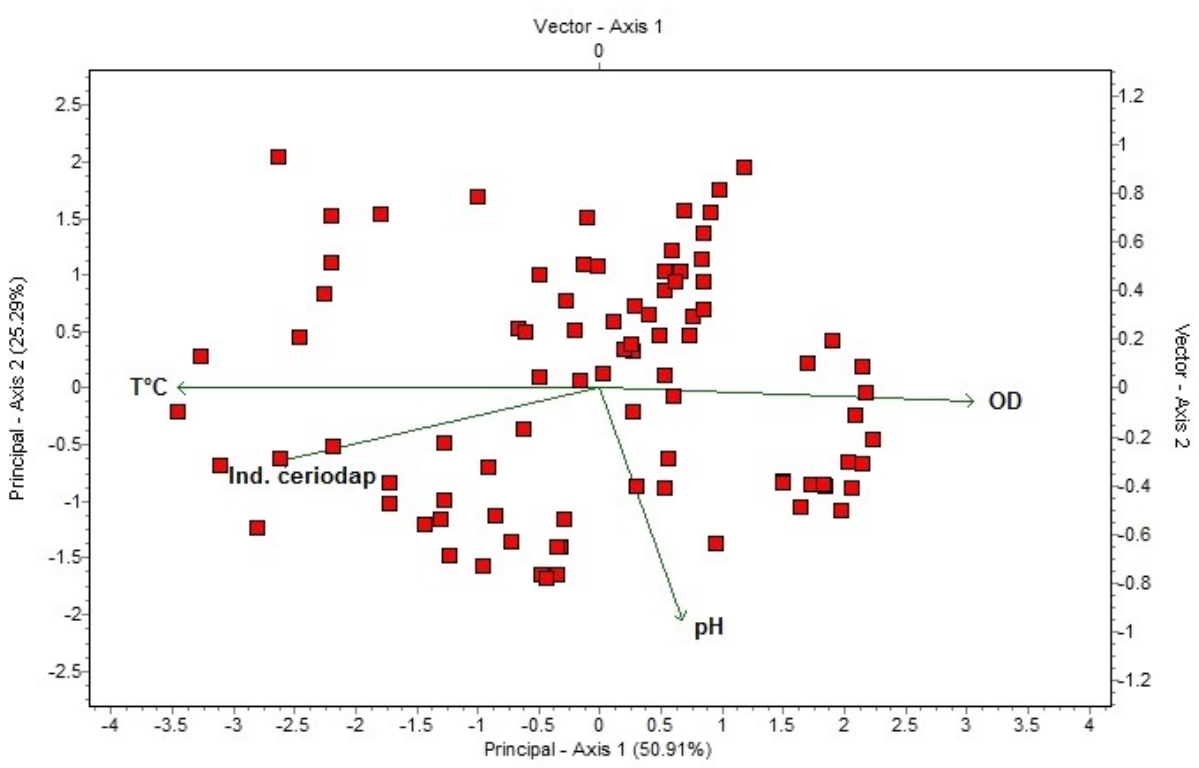

Figura 2. Análisis de componentes principales usando la matriz de correlación de parámetros de calidad de agua incluidas en el crecimiento de Ceriodaphnia sp en condiciones de laboratorio

enriquecimiento con avena soya. Rev Colomb Cienc Pec 23: 78-85.

15. Otero A, Muñoz M, MedinaVR, Cruz P. 2013. Efecto del alimento sobre variables productivas de dos especies de cladóceros bajo condiciones de laboratorio. Rev MVZ Córdoba 18: 36423647.

16. Peña-Aguado F, Nandini S, Sarma SSS. 2005. Differences in population growth of and cladocerans raised on algal diets supplemented with yeast. Limnologica 35: 298-303. doi: 10.1016/ j.limno.2005.08.002

17. Pennak R. 1978. Freshwater invertebrates of the United States. $2^{\text {nd }}$ ed. New York, USA: Wiley Interscience. 803 p.

18. Pérez C.1995. Cultivo experimental de diatomeas Thalassiosira subtilis, Skeletonema costatum y Chaetoceros affinis em condiciones de laboratório para fines de acuicultura. Tesis de Maestría. Manzanillo, Colombia: Univ. de Colina. $54 \mathrm{p}$.
19. Pérez I, García A, Garatachia M, Hernández M, Pérez C, Ortega L. 2015. Influencia de la temperatura y tipo de alimento en la historia de la vida de Ceriodaphnia cornuta Sars (1885) (Crustacea: Cladocera). Invest Cienc 64: 11-18.

20. Prieto M, Atencio V. 2008. Zooplancton en la larvicultura de peces neotropicales. Rev MVZ Cordoba 13: 1415-1425.

21. Prieto M, De la Cruz L, Morales M. 2006. Cultivo experimental del cladócero. Moina sp alimentado con Ankistrodesmus sp y Saccharomyces cerevisiae. Rev MVZ Córdoba 11: 705-714.

22. Rodríguez-Estrada J, VillaseñorCórdova R, Martínez-Jerónimo J. 2003. Efecto de la temperatura y tipo de alimento en el cultivo de Moina micrura (Kurz,1874) (Anopoda: Moìnidae) en condiciones de laboratorio. Hidrobiológica 13: 239-246.

23. Romero T. 2009. Desarrollo de Moina sp en condiciones de laboratorio alimentada con microalgas cultivadas en 
residuales pesqueros. REDVET 10(4). [Internet]. Disponible en: http:// www.veterinaria.org/revistas/redvet/ n040409.html

24. Romero TJ, Manso B, Martínez, F, Moreno M. 2010. Producción de Moina sp alimentada con Chlorella spp cultivada con riles orgánicos de la industria pesquera cubana. REDVET 11(12). [Internet]. Disponible en: https://www.redalyc.org/articulo.oa? $\mathrm{id}=63616936004$

25. Rottman RW, Graves JS, Watson C, Yanong RE. 2003. Culture techniques of Moina: the ideal Daphnia for feeding freshwater fish fry. Florida, USA: University of Florida. $6 \mathrm{p}$.

26. Santos M, Melao M, Lombardi AT. 2006. Life history characteristic and production of Ceriodaphnia silvestrii daday (Crustacea, Cladocera) under different experimental conditions. Acta Limnol Bras 18: 199-212.
27. Sipaúba-Tavares L, Rocha O. 2003. Produção de plancton (fitoplancton y zooplancton) para alimentação do organismos aquáticos. Brasil: Rima. $106 \mathrm{p}$.

28. Sipaúba-Tavares, Brachion MA. 2002. Population growth and development of two species of cladocera, Moina micrura y Diaphanosoma birgei, in laboratory. Braz J Biol 62: 701-711. doi: 10.1590/S1519-69842002000400018

29. Velu CS, Munuswany N. 2003. Nutritional evaluation of descapsulated cysts of fairy shrimp (Streptocephalus dichotomus) for ornamental fish larval rearing. Aquac Res 34: 967-974. doi: 10.1046/ j.1365-2109.2003.00961.x

30. Villalobos MJ, González EJ. 2005. Estudios sobre la biología y ecología de Ceriodaphnia cornuta SARS. Interciencia 31: 351-357. 